\title{
Serum markers thrombophilia in pregnant women with Systemic Lupus Erythematosus
}

Vanessa Marcon de Oliveira 1

Ernesto Antonio Figueiró-Filho 2

Cristiane Munaretto Ferreira 3

Erica Freire de Vasconcelos Pereira 4

1,3,4 Faculdade de Ciências Farmacêuticas, Alimentos e Nutrição. Universidade Federal de Mato Grosso do Sul. Caixa Postal 549. Cidade Universitária. Campo Grande, MS, Brasil. CEP: 79.0709-000. E-mail: vanamarcon@hotmail.com

2 Departamento de Ginecologia e Obstetricia. Universidade Federal de Mato Grosso do Sul. Campo Grande, MS, Brazil.

\begin{abstract}
Objectives: to determine the frequency of serum markers for hereditary and acquired thrombophilia and their association with pregnancy in women with Systemic Lupus Erythematosus (SLE).

Methods: a case-control study was conducted among 25 pregnant women with SLE (study group) and 32 pregnant women without known disease and with at least one previous pregnancy (control group). The presence of antiphospholipid antibodies and hereditary thrombophilia were examined in both groups. We used the $\chi^{2}$ Test with Yates correction or Fisher's Exact Test to verify the associations and calculate the relative risk.

Results: thrombophilia was present in $72.0 \%$ of pregnant women with SLE and in $6.0 \%$ of patients in the control group. A significant association was found between the presence of $S L E$ and serum markers for hereditary thrombophilia / antiphospholipid antibodies $(p<0.05)$. The relative risks for antiphospholipid antibodies were $13.20($ ICR95\%=1.81 - 96.46) in pregnant women with SLE, $7.26(C I 95 \%=1.77-29.86)$ for the presence of serum markers of hereditary thrombophilia and $7.92(C I 95 \%=2.62-3.94)$ for the presence of hereditary thrombophilia and/or antiphospholipid antibodies.

Conclusions: the identification of markers for hereditary and/or acquired thrombophilia in pregnant women with lupus may be clinically useful to determine which patients have a higher risk of obstetric complications.

Key words Systemic lupus erythematosus, Thrombophilia, Antiphospholipid syndrome, Pregnancy complications, hematologic
\end{abstract}




\section{Introduction}

Systemic lupus erythematosus (SLE) is a chronic autoimmune disease induced by multiple factors, characterized by the production of autoantibodies, which lead to the damage of multiple organs and changes in immunological patterns. ${ }^{1}$ It is a serious disease with significant mortality and morbidity, and approximately $90 \%$ of affected patients are women of childbearing age. ${ }^{2}$

The disease has a variety of signs and symptoms that are often unspecific, thereby making its diagnosis difficult. The diagnosis of SLE is made when at least four of the 17 criteria defined by the American College of Rheumatology for SLE are present (Table 1). 3
As the manifestations of a normal pregnancy may sometimes be confused with lupus activity, it is important to understand the changes that occur during pregnancy. 2 During normal pregnancy, changes occur in clotting factors, which result in protein $\mathrm{S}$ deficiency, increased fibrinogen levels, and changes in factor II and factor VII. These changes increase the risk of thromboembolism by five-fold. ${ }^{4}$ Thrombocytopenia is usually mild and does not cause bleeding problems. ${ }^{4}$

Thrombophilias are mainly related to the presence of antiphospholipid antibodies that are present in one-third of patients with SLE (currently a diagnostic criteria according to the American College of Rheumatology), and their presence is associated with poor pregnancy outcomes. 5 These antibodies

Table 1

Clinical and Immunologic Criteria for SLE.

Clinical Criteria

1) Acute cutaneous lupus

a) including lupus malar rash (do not count if malar discoid)

i) bullous lupus

ii) toxic epidermal necrolysis variant of SLE

iii) maculopapular lupus rash

iv) photosensitive lupus rash

(1) in the absence of dermatomyositis

b) or subacute cutaneous lupus

i) nonindurated psoriaform and/or annular polycyclic lesions that resolve without scarring, although occasionally with postinflammatory dyspigmentation or telangiectasias)

2) Chronic cutaneous lupus

a) including classical discoid rash

i) localized (above the neck)

ii) generalized (above and below the neck)

b) hypertrophic (verrucous) lupus

c) lupus panniculitis (profundus)

d) mucosal lupus

e) lupus erythematosus tumidus

f) chillblains lupus

g) discoid lupus/lichen planus overlap

3) Oral ulcers: palate

a) buccal

b) tongue

c) or nasal ulcers

d) in the absence of other causes, such as vasculitis, Behcets, infection (herpes), inflammatory bowel disease, reactive arthritis, and acidic foods

Criteria are cumulative and need not be present concurrently. Adapted from Petri et al.3 
Clinical and Immunologic Criteria for SLE.

\section{Clinical Criteria}

4) Nonscarring alopecia (diffuse thinning or hair fragility with visible broken hairs)

a) in the absence of other causes such as alopecia areata, drugs, iron deficiency and androgenic alopecia

5) Synovitis involving two or more joints, characterized by swelling or effusion OR tenderness in 2 or more joints and thirty minutes or more of morning stiffness.

6) Serositis

a) typical pleurisy for more than 1 day

i) or pleural effusions

ii) or pleural rub

b) typical pericardial pain (pain with recumbency improved by sitting forward) for more than 1 day

i) or pericardial effusion

ii) or pericardial rub

iii) or pericarditis by EKG

(1) in the absence of other causes, such as infection, uremia, and Dressler's pericarditis

7) Renal

a) Urine protein/creatinine (or $24 \mathrm{hr}$ urine protein) representing $500 \mathrm{mg}$ of protein/24 $\mathrm{hr}$ or

b) Red blood cell casts

8) Neurologic

a) seizures

b) psychosis

c) mononeuritis multiplex

i)in the absence of other known causes such as primary vasculitis

d) myelitis

e) peripheral or cranial neuropathy

i) in the absence of other known causes such as primary vasculitis, infection, and diabetes mellitus

f) acute confusional state

i) in the absence of other causes, including toxic-metabolic, uremia, drugs

9) Hemolytic anemia

10) Leukopenia ( $<4,000 / \mathrm{mm}^{3}$ at least once)

i)in the absence of other known causes such as Felty's, drugs, and portal hypertension

b) Lymphopenia $\left(<1,000 / \mathrm{mm}^{3}\right.$ at least once)

ii) in the absence of other known causes such as corticosteroids, drugs and infection

11)Thrombocytopenia $\left(<100,000 / \mathrm{mm}^{3}\right)$ at least once

a) in the absence of other known causes such as drugs, portal hypertension, and TTP

Immunological Criteria

1) ANA above laboratory reference range

2) Anti-dsDNA above laboratory reference range, except ELISA: twice above laboratory reference range

3) Anti-Sm

4) Antiphospholipid antibody: any of the following

a) lupus anticoagulant

b) false-positive RPR

c) medium or high titer anticardiolipin (IgA, IgG or $\lg \mathrm{M}$

d) anti- $\beta_{2}$ glycoprotein I (IgA, IgG or IgM)

5) Low complement
a) Iow C3
b) low $\mathrm{C} 4$
c) low $\mathrm{CH} 50$

6) Direct Coombs test in the absence of hemolytic anemia

Criteria are cumulative and need not be present concurrently. Adapted from Petri et al.3 
promote arterial and venous thrombosis, thrombocytopenia and pregnancy loss.6,7 In despite of these information, previous reports regarding hereditary thrombophilia serum markers are addressed in nonpregnant women with SLE. ${ }^{8-10}$

Large numbers of complications can occur in pregnant women with SLE. Possible adverse events include reactivation of the disease, thrombosis and miscarriage in women with antiphospholipid antibodies, neonatal lupus, hypertension and toxicity of drugs used to treat SLE. 11 The high disease activity during pregnancy results in an increased frequency of preterm births and a decreased likelihood of livebirth, with almost $25 \%$ of pregnancies resulting in fetal death. ${ }^{12}$ It is recommended that the immunosuppressive drugs needed to control SLE continue to be administered, particularly if the patient has major organ involvement, such as lupus nephritis.12 Corticosteroids are relatively safe for use during pregnancy, therefore it may contribute in increasing maternal hypertension and gestational diabetes. Prednisone is the choice drug option in cases of active SLE disease upon the maternal organism, for it does not cross the placental barrier. By the other hand, dexamethasone should enter fetal circulation, so that it becomes the best choice in treatment of congenital heart block. ${ }^{2}$

The objective of the present study was to determine the frequency of serum markers for hereditary and acquired thrombophilia and antiphospholipid antibodies and to examine their association with pregnancy in women with SLE.

\section{Methods}

We carried out a prospective, observational, crosssectional controlled study in pregnant women from the Service of Gynecology and Obstetrics at the Hospital University Maria Aparecida Pedrossian (HUMAP) from June 2007 to June 2012. These were two distinct groups of patients, who were classified according to the following criteria:

Control Group (CG) - consisted of pregnant women without known disease who had at least one previous pregnancy, whose previous pregnancies were all low-risk and resulted in term deliveries and who had no history of miscarriage. Patients with a prior history of serum markers of hereditary thrombophilias and antiphospholipid antibodies were excluded from this group.

Study Group (SG) - consisted of pregnant women with a diagnosis of Systemic Lupus Erythematosus (SLE) prior to pregnancy based on clinical and laboratory criteria ${ }^{3}$ (Table 1 ) who also might not have been diagnosed with hereditary thrombophilia or antiphospholipid antibodies prior to the current pregnancy.

Pregnant women whose SLE was in the active phase, who were less than 18 years of age and those whose diagnosis of SLE was made in the current pregnancy were excluded from the study.

The selection of patients was conducted prospectively and at random according to the order in which cases meeting the inclusion criteria of the outpatient high-risk pregnancy (SG) group and the low-risk (CG) group presented during the study period.

The calculation of the simple random sampling minimum number of pregnant women with a prior history of SLE to be included in SG took the following factors into account: the total population of primary care patients at the Clinic of High-Risk Pregnancy HUMAP (1,000 pregnant patients/year); the prevalence of antiphospholipid antibodies in this group of patients, whose values are well established in the literature and are approximately $40 \%, 13$ the prevalence of thrombophilia in the general population, which is approximately $5 \%, 14$ and test power of $85 \%$ and a sampling error of $5 \%$ using a two-tailed test. With these parameters, the minimum number of pregnant women required in both groups was 24 .

All patients were screened for the presence of antiphospholipid antibodies by serum IgM and IgG anticardiolipin, lupus anticoagulant and anti- $\beta_{2}$ glycoprotein I (anti- $\left.\beta_{2} \mathrm{GPI}\right)$. In despite of the literature recommendation of repeating the dosage within 12 weeks interval, it was not performed due to specific characteristics of our routine service. 12

Pregnant women in whose blood sample the antiphospholipid antibodies were identified, were considered as antiphospholipid serum markers carriers, hence they were not classified as Antiphospholipid Antibody Syndrome (APS) disease carriers. The presence of hereditary thrombophilia was identified through the measurement of protein $\mathrm{C}$ and $\mathrm{S}$ coagulation, antithrombin, homocysteine and research Q506 mutation of factor V (factor V Leiden). Among pregnant women in the study group, the measurements were performed at the first prenatal consultation of the current pregnancy, this visit was prior to 20 weeks gestation. Among pregnant women in the control group who met the inclusion criteria, samples were collected in the immediate postpartum period (up to six hours postpartum) to avoid sample loose. The screening for acquired/ inherited thrombophilia is routinely performed at the Clinic of High-Risk Pregnancy at our institution. The serum levels of factor VIII, prothrombin G20210A mutation, and enzyme methylenetetrahy- 
drofolate reductase (MTHFRC677T) polymorphism are not screened as routine in our service.

The anticardiolipin antibodies IgM and IgG were processed using enzyme immunoassay (ELISA) following the methodology proposed in international standardization. 15 The results were considered reactive for IgM and $\operatorname{IgG}$ when they had values higher than 11 UMPL and 11 UGPL, respectively.

The presence of lupus anticoagulant was conducted in three stages, and the first test was performed with the screening techniques aPTT (activated partial thromboplastin time) and dRWT (time of the dilute Russell viper venom). When the obtained values were greater than 1.26 and/or 1.14 in the aPTT tests and dRWT, respectively, they were held at the second step, where the aPTT test proceeded using a mixture of $50 \%$ plasma and $50 \%$ of the patient's normal plasma. If no correction was needed, the test proceeded to the third stage. This consisted of dRWT confirmatory testing, which tested positive for lupus anticoagulant when the concentration was greater than 1.21 .15

The search for anti- $\beta_{2}$ GPI antibodies was performed by ELISA, where values above $15 \mathrm{U} / \mathrm{mL}$ were considered to be reactive. 15

The dosage of protein $\mathrm{C}$ was measured using the chromogenic method, 16,17 the result of which is the finding of a normal serum value greater than or equal to $65 \%$. Values less than $65 \%$ are considered to indicate protein $\mathrm{C}$ deficiency. 16,17

The dosage of protein $\mathrm{S}$ was measured using the chromogenic method, 16,17 the result of which is the finding of a normal serum value greater than or equal to $55 \%$. Values below $30 \%$ are considered to indicate protein $\mathrm{S}$ deficiency. 16,17

The dosing of antithrombin was measured using the chromogenic method, 16,17 the result of which is the finding of a normal serum value of greater than or equal to $60 \%$. Values below $60 \%$ are considered to indicate antithrombin deficiency. 16,17

Homocysteine was measured using high-pressure liquid chromatography (HPLC) with isocratic elution and fluorimetric detection, 18 whose reference value is 4 to $12 \mu \mathrm{mol} / \mathrm{L}$. Hyperhomocysteinemia is evidenced by values greater than $12 \mu \mathrm{mol} / \mathrm{L} .18$

The research Q506 mutation in the factor $\mathrm{V}$ (factor V Leiden) was measured using polymerase chain reaction (PCR) using to the restriction enzyme Mnli,16 and is considered to be a positive mutation (homozygous or heterozygous ).

Carriers of hereditary thrombophilia were all pregnant women who had test results that indicated protein $\mathrm{C}$ deficiency, protein $\mathrm{S}$ deficiency, antithrombin deficiency, hyperhomocysteinemia or the presence of Factor V Leiden mutation (heterozygous or homozygous affected). Carriers of antiphospholipid antibodies were all patients whose results were positive for anticardiolipin IgM/IgG, lupus anticoagulant and/or anti- $\beta_{2}$ GPI, according to the methodology explained.

We emphasize that serum markers for acquired thrombophilias were not repeated after 12 weeks, because the present study did not aim to perform the diagnosis of APS.

The parametric variables (age and parity) are expressed as the mean \pm standard deviation and were compared using the Student $t$ test. The nonparametric variables (presence/absence of hereditary thrombophilia and antiphospholipid antibodies) were evaluated in double-entry contingency tables using the $\chi^{2}$ test with Yates correction or Fisher's Exact Test. Significant associations were those with $p$ value $<0.05$. Relative risks were calculated and are presented with $95 \%$ confidence intervals (CI).

The patients included in the study signed an informed consent form (ICF). The study and its ICF were approved by the Ethics Committee on Human Research of the Federal University of Mato Grosso do Sul - UFMS, Protocol 884, as of May 18, 2007.

\section{Results}

Of the 57 pregnant women studied, $25(43.9 \%)$ were in the study group and $32(56.1 \%)$ were in the control group. The mean age in the SG was 29.4 years, and the average number of pregnancies was $2.1 \pm 1.4$. In the $C G$, the mean age was $30.3 \pm 4.4$ years, and the mean number of pregnancies was $1.2 \pm 0.4$. No difference between the groups was found for maternal age or parity.

Positive screening for hereditary thrombophilia and/or antiphospholipid antibodies was found in $72.0 \%(18 / 25)$ of pregnant women in the SG. Among the CG patients, the presence of thrombophilia and/or antiphospholipid antibodies was found in $9.4 \%(3 / 32)$ of the sample (Table 2$)$.

Screening for antiphospholipid antibodies in pregnant women with lupus demonstrated positive results in ten women (40\%), out of these, six (24\%) patients were identified with isolated antibodies and $4(16 \%)$ patients had antibodies associated with other thrombophilia serum markers. In the pregnant women from the CG the presence of antiphospholipid antibodies was detected in only one patient (3.1\%) (Table 2).

There was an association $(p<0.05)$ between the presence of SLE in pregnant women and the occurrence of serum markers for hereditary thrombophilia 
Table 2

Association between the presence of SLE and the occurrence of serum markers of thrombophilias in pregnant women.

\begin{tabular}{|c|c|c|c|c|c|c|c|c|c|c|c|c|c|c|c|c|c|c|}
\hline & \multicolumn{6}{|c|}{$\begin{array}{l}\text { Serum markers of antiphospholipid } \\
\text { antibodies* }\end{array}$} & \multicolumn{6}{|c|}{$\begin{array}{l}\text { Serum markers of hereditary } \\
\text { thrombophilia }\end{array}$} & \multicolumn{6}{|c|}{$\begin{array}{c}\text { Serum markers of hereditary } \\
\text { thrombophilia and/or antiphospholipid } \\
\text { antibodies }\end{array}$} \\
\hline & \multicolumn{2}{|c|}{ Yes } & \multicolumn{2}{|c|}{ No } & \multicolumn{2}{|c|}{ Total } & \multicolumn{2}{|c|}{ Yes } & \multicolumn{2}{|c|}{ No } & \multicolumn{2}{|c|}{ Total } & \multicolumn{2}{|c|}{ Yes } & \multicolumn{2}{|c|}{ No } & \multicolumn{2}{|c|}{ Total } \\
\hline & $\mathrm{n}$ & $\%$ & $\mathrm{n}$ & $\%$ & $\mathrm{n}$ & $\%$ & $\mathrm{n}$ & $\%$ & $n$ & $\%$ & $n$ & $\%$ & $\mathrm{n}$ & $\%$ & $n$ & $\%$ & $\mathrm{n}$ & $\%$ \\
\hline Study group 1 & 10 & 40.0 & 15 & 60.0 & 25 & 100.0 & 11 & 44.0 & 14 & 56.0 & 25 & 100.0 & 18 & 72.0 & 7 & 28.0 & 25 & 100.0 \\
\hline Control group 2 & 1 & 3.1 & 31 & 96.9 & 32 & 100.0 & 2 & 6.3 & 30 & 93.7 & 32 & 100.0 & 3 & 9.4 & 29 & 90.6 & 32 & 100.0 \\
\hline Total & 11 & 19.3 & 46 & 80.7 & 57 & 100.0 & 13 & 22.8 & 44 & 77.2 & 57 & 100.0 & 21 & 36.8 & 36 & 63.2 & 57 & 100.0 \\
\hline RR (IC95\%) & \multicolumn{6}{|c|}{$13.20(1.81-96.46)$} & \multicolumn{6}{|c|}{$7.26(1.77-29.86)$} & \multicolumn{6}{|c|}{$7.92(2.62-23.94)$} \\
\hline$P$ & \multicolumn{6}{|c|}{0.001} & \multicolumn{6}{|c|}{0.001} & \multicolumn{6}{|c|}{$<0.001$} \\
\hline
\end{tabular}

*Fisher's Exact Test; 1pregnant women with SLE; 2pregnant women with at least one successful pregnancy. 
Frequency of serum markers for thrombophilias in pregnant women with and without SLE.

\begin{tabular}{|c|c|c|}
\hline $\begin{array}{l}\text { Hereditary thrombophilia and / or antiphospholipid } \\
\text { antibodies }\end{array}$ & $\begin{array}{l}\text { Study Group } 1 \\
\% \text { (n/total group) }\end{array}$ & $\begin{array}{l}\text { Control Group } 2 \\
\%(n / \text { total group) }\end{array}$ \\
\hline Isolated antiphospholipid antibodies & $24.0 \%(6 / 25)$ & $3.1 \%(1 / 32)$ \\
\hline Antibody IgM anticardiolipin & $4.0 \%(1 / 25)$ & $3.1 \%(1 / 32)$ \\
\hline Lupus anticoagulant & $20.0 \%(5 / 25)$ & $-(0 / 32)$ \\
\hline Isolated hereditary thrombophilia & $24.0 \%(6 / 25)$ & $6.2 \%(2 / 32)$ \\
\hline Protein C Deficiency & $4.0 \%(1 / 25)$ & $-(0 / 32)$ \\
\hline Protein S Deficiency & $4.0 \%(1 / 25)$ & $3.1 \%(1 / 32)$ \\
\hline Antithrombin Deficiency & $12.0 \%(3 / 25)$ & $3.1 \%(1 / 32)$ \\
\hline Hyperhomocysteinemia & $4.0 \%(1 / 25)$ & $-(0 / 32)$ \\
\hline $\begin{array}{l}\text { Hereditary thrombophilia and / or antiphospholipid antibodies } \\
\text { identified together }\end{array}$ & $24.0 \%(6 / 25)$ & $-(0 / 32)$ \\
\hline Anticardiolipin antibody $\lg M+$ antithrombin deficiency & $4.0 \%(1 / 25)$ & $-(0 / 32)$ \\
\hline Anticardiolipin antibody IgG + antibody anti- $\beta_{2} \mathrm{GPI}$ & $4.0 \%(1 / 25)$ & $-(0 / 32)$ \\
\hline Lupus anticoagulant + antithrombin deficiency & $8.0 \%(2 / 25)$ & $-(0 / 32)$ \\
\hline Deficiency of protein $S+$ deficiency of antithrombin & $8.0 \%(2 / 25)$ & $-(0 / 32)$ \\
\hline
\end{tabular}

1 pregnant women with SLE; 2pregnant women with at least one successful pregnancy.

and/or antiphospholipid antibodies (Table 2).

The relative risk for the presence of antiphospholipid antibodies in pregnant women with SLE was $13.20(\mathrm{CI} 95 \%=1.81-96.46)$. The presence of serum markers for hereditary thrombophilia had a relative risk of $7.26(\mathrm{CI} 95 \%=1.77-29.86)$. The relative risk was $7.92(\mathrm{CI} 95 \%=2.62-23.94)$ when considering the occurrence of serum markers for hereditary thrombophilia and/or antiphospholipid antibodies.

Isolated hereditary thrombophilia was present in $24.0 \%$ of the pregnant women diagnosed with SLE $(6 / 25)$ and $6.1 \%(2 / 32)$ of pregnant women in control group. Two or more hereditary thrombophilias and / or the presence of antiphospholipid antibodies together were found in 24\% (6/25) of patients EG (Table 3). Among pregnant women in the SG, the most frequent isolated hereditary thrombophilias were as follows: antithrombin deficiency (12\%), protein $\mathrm{C}$ and $\mathrm{S}$ deficiencies, and hyperhomocysteinemia (4\%). In screening for the presence of antiphospholipid antibodies, the presence of lupic anticoagulant alone was demonstrated in $20 \%$ of the sample group $(5 / 25)$ (Table 3$)$.

\section{Discussion}

The prevalence of thrombophilic factors in pregnant women with SLE is not well established in the literature. Thus, the investigation of the prevalence of these factors in this group of patients helps to clarify its importance both in these clinical profiles and in preventing obstetric complications. The presence of antiphospholipid antibodies in patients with SLE has been described,11,13,19 but markers for hereditary thrombophilia are not well characterized, and varying methodologies make it difficult to compare data. $8-10$

The prevalence of antiphospholipid antibodies in SLE patients is approximately $40 \%$, and between $12 \%$ and $44 \%$ of patients are positive for anticardiolipin, $15 \%$ to $34 \%$ are positive for lupus anticoagulant and $10 \%$ to $19 \%$ are positive for the anti- $\beta_{2}$ glycoprotein I ( $\beta_{2}$-GPI anti). 13 These data agree with the results of the present study, where $40 \%(10 / 25)$ of patients had markers for antiphospholipid antibodies. Anticardiolipin antibody was found in $12 \%$ $(3 / 25)$ of patients, lupus anticoagulant was found in $24 \%(6 / 25)$ and anti- $\beta_{2}$ GPI was found in $4 \%(1 / 25)$, which is the only antibody whose value was below that described in the literature.

Thrombotic events occur more frequently in lupus patients with antiphospholipid antibodies than in patients without SLE or other autoimmune disease who have these antibodies. ${ }^{20}$ A study conducted in 2009 showed similar results and found a higher frequency of thrombosis and pregnancy loss in patients with SLE, which were associated with the presence of markers for thrombophilia in patients with acquired primary APS. 21

The presence of markers of antiphospholipid 
antibodies was found to be 3.1 times higher in women experiencing pregnancy loss, especially after 20 weeks gestation. This was an independent risk factor for additional losses in a pregnancy cohort study of 166 pregnant women in the Hopkins Lupus Center.22

Maternal lupus activity and the presence of antiphospholipid antibodies were associated with concomitant major causes of obstetric complications, ${ }^{12,22}$ It is estimated that approximately $20 \%$ of pregnancies in women with SLE result in fetal loss. 23 The rate of preterm birth (birth before 37 weeks gestation) is also increased in this group of patients. The incidence appears to vary between $23 \%$ and $28 \% .23$ Preterm birth is often spontaneous, and it is mainly due to premature rupture of membranes, but there is also a significant portion of cases in which labor is induced to protect the health of mother and / or baby (early fetal distress or preeclampsia). 19

In relation to serum markers of hereditary thrombophilia, protein $\mathrm{C}$ deficiency, $\mathrm{S}$ and antithrombin, we found an incidence of $40 \%$ (10/25) among pregnant women with lupus, however several authors, ${ }^{9,24,25}$ did not find the presence of these markers in non-pregnant patients with SLE, suggesting that they were not associated with an increased thrombotic risk. However, we observed changes in these protein systems. 10

We cannot say that patients with protein $\mathrm{C}$ and $\mathrm{S}$ deficiency are carriers of inherited deficiency since pregnancy is a hypercoagulable state with increased coagulation factors and simultaneous decrease of natural anticoagulant and fibrinolytic proteins. These important changes occur naturally in order to minimize the risk blood loss, but increases the occurrence of thromboembolic events. However as there were no subsequent dosages of natural anticoagulants, it is suggested that these deficiencies may be transient.26-28

We did not observe the presence of FV Leiden mutation in any patient. However, Brouwer et al. ${ }^{24}$ found that FV Leiden in non-pregnant patients with
SLE increases the risk of venous thrombosis and is associated with an OR of 3.5.

The presence of hyperhomocysteinemia was found in only $4 \%(1 / 25)$ of women in the study group, a result which is lower than the one presented in the literature in non-pregnant patients with lupus, which is approximately $37 \%$, being this related to renal impairment8. High concentrations of this amino acid are common in SLE patients and are predictive of late thrombosis, stroke and hypertension. 21

Studies show that women with thrombophilia (protein $\mathrm{S}$ deficiency, and antithrombin $\mathrm{C}$, hyperhomocysteinemia and factor $\mathrm{V}$ Leiden mutation) during pregnancy have a higher prevalence of obstetric and perinatal complications such as miscarriage, fetal loss and preeclampsia.6,7 Thus, the high incidence of these markers in women with lupus may be related to the increased number of cases of obstetric complications in this group of women.

Whereas women with SLE have 2 to 4 times higher rates of complications during pregnancy than non-pregnant women with SLE, 29 the identification of markers for hereditary and/or acquired thrombophilia in pregnant women with lupus may be clinically useful to determine which patients have a higher risk of obstetric complications.

We emphasize that our study population portrays some characteristics which make it quite different from the populations studied by other authors. The Brazilian racial miscegenation could induce genetic characteristics not found in populations studied in other trials regarding hereditary/acquired thrombophilia. 29,30

We either did not find any study that focused in the association of SLE pregnant women and inherited thrombophilia. We were only able to find data on the prevalence of acquired thrombophilia (antiphospholipid antibodies) and its impact on pregnancy in patients with SLE. This may suggest that more studies regarding the impact of inherited thrombophilia in pregnant women with SLE should be carried on.

\section{References}

1. Wei Q, Ouyang Y, Zeng W, Duan L, Ge J, Liao H Pregnancy complicating systemic lupus erythematosus: a series of 86 cases. Arch Gynecol Obstet. 2011; 284 (5): 1067-71.

2. Dhar JP, Sokol RJ. Lupus and pregnancy: complex yet manageable. Clin Med Res. 2006; 4 (4): 310-21.

3. Petri M, Orbai AM, Alarcón GS, Gordon C, Merrill JT,
Fortin PR, Bruce IN, Isenberg D, Wallace DJ, Nived O, Sturfelt G, Ramsey-Goldman R, Bae SCV, Hanly JG, Sanchez-Guerrero J, Clarke A, Aranow C, Manzi S, Urowitz M, Gladman D, Kalunian K, Costner M, Werth VP, Zoma A, Bernatsky S, Ruiz-Irastorza G, Khamashta MA, Jacobsen S, Buyon JP, Maddison P, Dooley MA, van Vollenhoven RF, Ginzler E, Stoll T, Peschken C, Jorizzo JL, 
Callen JP, Lim SS, Fessler BJ, Inanc M, Kamen DL, Rahman A, Steinsson K, Franks Jr. AG, Sigler L, Hameed S, Fang H, Pham N, Brey R, Weisman MH, McGwin Jr. G, Magder LS. Derivation and Validation of Systemic Lupus International Collaborating Clinics Classification Criteria for Systemic Lupus Erythematosus. Arthritis Rheum. 2012; 64 (8): 2677-86.

4. Dresang LT, Fontaine P, Leeman L, King VJ. Venous thromboembolism during pregnancy. Am Fam Physician. 2008; 77 (12): 1709-16.

5. Zhan Z, Yang Y, Zhan Y, Chen D, Liang L, Yang X. Fetal outcomes and associated factors of adverse outcomes of pregnancy in southern Chinese women with systemic lupus erythematosus. PLoS One. 2017; 12 (4): e0176457.

6. Figueiró-Filho E, Oliveira V. Association of recurrent abortion, fetal loss and severe preeclampsia with hereditary thrombophilias and antiphospholipid antibodies in pregnant women of central Brazil. Rev Bras Ginecol Obstet. 2007; 29 (11): 561-7

7. Figueiro-Filho EA, Oliveira VM, Coelho LR, Breda I. Serum markers of inherited thrombophilia and antiphospholipid antibodies in pregnant women with previous history of severe pre-eclampsia. Rev Bras Ginecol Obstet. 2012; 34 (1): 40-6

8. Barcat D, Guérin V, Ryman A, Constans J, Vernhes JP, Vergnes C, Bonnet F, Delbrel X, Morlat P, Longy-Boursier $\mathrm{M}$, Conri C. Thrombophilia and thrombosis in systemic lupus erythematosus: a case-control study. Ann Rheum Dis 2003; 62 (10): 1016-7.

9. Afeltra A, Vadacca M, Conti L, Galluzzo S, Mitterhofer AP, Ferri GM, Del Porto F, Caccavo D, Gandolfo GM, Amoroso A. Thrombosis in systemic lupus erythematosus: congenital and acquired risk factors. Arthritis Rheum. 2005; 53 (3): 452-9

10. Vayá A, Santaolaria M, Micó L, Calvo J, Oropesa R, Villa P, Todoli J, Simó M, Corella D, Ricart JM Thrombotic events in systemic lupus erythematosus. Its association with acquired and inherited thrombophilic defects. Clin Hemorheol Microcirc. 2008; 40 (2): 79-87.

11. Manzano-Gamero V, Pardo-Cabello AJ, Vargas-Hitos JA, Zamora-Pasadas M, Navarrete-Navarrete N, Sabio JM, Jáimez-Gámiz L, Ríos-Fernandez R, Ortego-Centeno N, Ayala-Gutierrez MM, de Ramón E, Colodro-Ruíz A, MicóGiner L, Castillo-Palma MJ, Robles-Marhuenda Á, LunaDel Castillo JD, Jiménez-Alonso J; Spanish Autoimmune Diseases Study Group (GEAS). Effect of ethnicity on clinical presentation and risk of antiphospholipid syndrome in Roma and Caucasian patients with systemic lupus erythematosus: a multicenter cross-sectional study. Int J Rheum Dis. 2017; 7. [Epub ahead of print]

12. Clowse ME, Magder LS, Witter F, Petri M. The impact of increased lupus activity on obstetric outcomes. Arthritis Rheum. 2005; 52 (2): 514-21.

13. Biggioggero M, Meroni PL. The geoepidemiology of the antiphospholipid antibody syndrome. Autoimmun Rev. 2010; 9 (5): A299-304

14. Middeldorp S, van Hylckama Vlieg A. Does thrombophilia testing help in the clinical management of patients? Br J Haematol. 2008; 143 (3): 321-35.
15. Miyakis S1, Lockshin MD, Atsumi T, Branch DW, Brey RL, Cervera R, Derksen RH, DE Groot PG, Koike T, Meroni PL, Reber G, Shoenfeld Y, Tincani A, Vlachoyiannopoulos PG, Krilis SA. International consensus statement on an update of the classification criteria for definite antiphospholipid syndrome (APS). J Thromb Haemost. 2006; 4 (2): 295-306.

16. Jackson BR, Holmes K, Phansalkar A, Rodgers GM. Testing for hereditary thrombophilia: a retrospective analysis of testing referred to a national laboratory. BMC Clin Pathol. 2008. 2; 8: 3.

17. Abbassi-Ghanavati M, Greer LG, Cunningham FG. Pregnancy and laboratory studies: a reference table for clinicians. Obstet Gynecol. 2009; 114 (6): 1326-31.

18. Ubbink JB, Hayward Vermaak WJ, Bissbort S. Rapid highperformance liquid chromatographic assay for total homocysteine levels in human serum. J Chromatogr. 1991; 565 (1-2): 441-6.

19. Andreoli L, Fredi M, Nalli C, Reggia R, Lojacono A, Motta M, Tincani A. Pregnancy implications for systemic lupus erythematosus and the antiphospholipid syndrome. J Autoimmun. 2012; 38 (2-3): J197-208.

20. Laskin CA, Clark CA, Spitzer KA. Antiphospholipid syndrome in systemic lupus erythematosus: is the whole greater than the sum of its parts? Rheum Dis Clin North Am. 2005; 31 (2): 255-72.

21. Danowski A, de Azevedo MN, de Souza Papi JA, Petri M. Determinants of risk for venous and arterial thrombosis in primary antiphospholipid syndrome and in antiphospholipid syndrome with systemic lupus erythematosus. J Rheumatol. 2009; 36 (6): 1195-9.

22. Clowse ME, Magder LS, Witter F, Petri M. Early risk factors for pregnancy loss in lupus. Obstet Gynecol. 2006; 107 (2 Pt 1): 293-9.

23. Yan Yuen S, Krizova A, Ouimet JM, Pope JE. Pregnancy Outcome in Systemic Lupus Erythematosus (SLE) is Improving: Results from a Case Control Study and Literature Review. Open Rheumatol J. 2008; 2: 89-98

24. Brouwer JL, Bijl M, Veeger NJ, Kluin-Nelemans HC, van der Meer J. The contribution of inherited and acquired thrombophilic defects, alone or combined with antiphospholipid antibodies, to venous and arterial thromboembolism in patients with systemic lupus erythematosus. Blood. 2004; 104 (1): 143-8.

25. Torresan M, Machado TF, Siqueira LH, Ozelo MC, Arruda VR, Annichino-Bizzacchi JM. The impact of the search for thrombophilia risk factors among antiphospholipid syndrome patients with thrombosis. Blood Coagul Fibrinolysis. 2000; 11 (7): 679-82.

26. Adachi T. Protein S and congenital protein S deficiency: the most frequent congenital thrombophilia in Japanese. Curr Drug Targets. 2005; 6 (5): 585-92.

27. Paidas MJ, Ku DH, Lee MJ, Manish S, Thurston A, Lockwood CJ, Arkel YS. Protein Z, protein S levels are lower in patients with thrombophilia and subsequent pregnancy complications. J Thromb Haemost. 2005; 3 (3): 497 501. 
28. Hojo S, Tsukimori K, Kinukawa N, Hattori S, Kang D, Hamasaki N, et al. Decreased maternal protein $\mathrm{S}$ activity is associated with fetal growth restriction. Thromb Res. 2008; 123 (1): 55-9.

29. Clowse ME, Jamison M, Myers E, James AH. A national study of the complications of lupus in pregnancy. Am J Obstet Gynecol. 2008; 199 (2): 127 e1-6.

Received on March 20, 2017

Final version presented on July 10, 2017

Approved on October 5, 2017
30. De Stefano V1, Martinelli I, Rossi E, Battaglioli T, Za T, Mannuccio Mannucci P, Leone G. The risk of recurrent venous thromboembolism in pregnancy and puerperium without antithrombotic prophylaxis. Br J Haematol. 2006; 135 (3): 386-91. 